\title{
Nht1, a Transposable Element Cloned from a Dispensable Chromosome in Nectria haematococca
}

\author{
Jürg Enkerli, ${ }^{1}$ Garima Bhatt, ${ }^{2}$ and Sarah F. Covert ${ }^{2}$ \\ ${ }^{1}$ Department of Botany, and ${ }^{2}$ Daniel B. Warnell School of Forest Resources, University of Georgia, Athens \\ 30602, U.S.A. \\ Received 28 January 1997. Accepted 23 April 1997.
}

\begin{abstract}
Certain isolates of the plant-pathogenic fungus Nectria haematococca mating population VI (MPVI) contain dispensable chromosomes that are unstable during sexual reproduction. Several of these chromosomes carry genes for phytoalexin detoxification and thus contribute to the pathogenic potential of this organism. A repeated DNA sequence, Nht1, was cloned from one of these dispensable chromosomes in $N$. haematococca MPVI. One copy of the repeated element (Nht1A) was completely sequenced. It is 2,198 bp long and it possesses incomplete inverted terminal repeats (ITRs) at each end. Nht1B, a partially sequenced copy of Nht1, has complete ITRs. Nht1A appears to contain 2 introns and encodes a protein of $\mathbf{5 5 0}$ amino acids that is highly similar to the protein encoded by the Fusarium oxysporum transposon, Fot1. Due to the presence of ITRs, its repeated nature, and its similarity to Fot1, we conclude that Nht1 is a transposable element. Within North American N. haematococca MPVI populations, Nht1 is distributed discontinuously. Its copy number in different field isolates varies from zero to approximately 100 copies per genome. The Nht1A source isolate is estimated to contain nine to 11 copies of $N$ ht1; at least six are on the chromosome from which Nht1A was cloned.
\end{abstract}

Additional keywords: chromosome-length polymorphisms, Fusarium solani.

Nectria haematococca mating population VI Berk. \& Broome (Fusarium solani f. sp. pisi) is a plant-pathogenic, filamentous ascomycete with a wide host range. It is able to infect monocot species such as maize and barley, as well as dicot species such as chickpea, garden pea, and red clover. Its interaction with chickpea (Cicer arietinum) and garden pea (Pisum sativum) has been studied intensely with respect to the importance of phytoalexin detoxification as a virulence determinant (VanEtten et al. 1989a; Miao and VanEtten 1992b). One gene $(M A K 1)$ encoding detoxification of maackiain, a chickpea phytoalexin, and six genes (PDAl to PDA6) encoding detoxification of pisatin, a pea phytoalexin, have been cloned from $N$. haematococca mating population VI (MPVI) (Weltring et al. 1988; VanEtten et al. 1989b; Reimmann and

Corresponding author: Sarah F. Covert

E-mail: Covert@BSCR.UGA.EDU

Nucleotide and/or amino acid sequence data are to be found at GenBank as accession number U78574.
VanEtten 1994; Covert et al. 1996). Transformation-mediated addition of PDA to $\mathrm{Pda}^{-}$strains (Ciuffetti and VanEtten 1996) and disruption of PDAl (Wasmann and VanEtten 1996) both indicate that highly expressed $P D A$ genes contribute to the virulence of $N$. haematococca MPVI on garden pea.

All of the known phytoalexin detoxification genes in $N$. haematococca MPVI are located on dispensable chromosomes (VanEtten et al. 1994). These chromosomes can be lost from the genome without affecting saprophytic growth, and do not hybridize to the essential portion of the genome when used to probe Southern blots of pulsed field gels (Miao et al. 1991a). Evidence for the presence of additional pathogenicity genes on the PDAl chromosome was recently published. Loss of this particular chromosome (Wasmann and VanEtten 1996) or deletion of $100 \mathrm{~kb}$ from this chromosome (Kistler et al. 1996) leads to nearly complete loss of virulence on garden pea. It appears, therefore, that dispensable chromosomes are reservoirs for pathogenicity/host specificity genes in this organism. Dispensable chromosomes have also been reported in at least three other fungal species but their role in pathogenicity, if any, remains uncertain (Tzeng et al. 1992; Masel et al. 1993b; Orbach et al. 1996). Knowledge of dispensable chromosome structure and organization is very limited. In addition to $M A K 1$ and the PDA genes, the only fungal gene to be cloned from a dispensable chromosome is a cyclin homologue in Colletotrichum gloeosporioides (Masel et al. 1993c). A few molecular markers specific to dispensable chromosomes have also been reported (Tzeng et al. 1992; Masel et al. 1993a; Orbach et al. 1996).

$N$. haematococca MPVI isolate $156-30-6$ has a $1.6-\mathrm{Mb}$ dispensable chromosome that carries the MAKI and PDA6-1 genes (Miao et al. 1991a). Genetic evidence suggests that $M A K 1$ contributes to the virulence of this isolate on chickpea (Miao and VanEtten 1992b). PDA6-1, however, does not appear to contribute to pathogenicity on pea (VanEtten et al. 1989b). In addition to being dispensable, the MAK1 PDA6-1 chromosome is unstable during sexual reproduction. In progeny analyzed from two crosses, this chromosome was frequently lost or converted into nonparental-sized chromosomes (Miao et al. 1991a). The generation of nonparental chromosomes during sexual reproduction has also been reported in other fungi, including Coprinus cinereus (Zolan et al. 1994) and Leptosphaeria maculans (Plummer and Howlett 1993). Although the mechanisms underlying these chromosomal rearrangements have not been elucidated, it has been proposed that dispersed repeated sequences play a critical role by serv- 
ing as sites for recombination within or between chromosomes (Zolan 1995).

Transposable elements are a common type of dispersed repeated sequence in fungal genomes. They are classified according to their mechanism of transposition (Finnegan 1989). Class I elements transpose via RNA intermediates and may or may not be flanked by direct terminal repeats. Class II elements transpose via a DNA-DNA mediated mechanism and are defined by inverted terminal repeats (ITRs). In recent years, members of both classes of transposable elements have been found in several different filamentous fungi (Kinsey and Helber 1989; Dobinson et al. 1993; Daboussi and Langin 1994; Kachroo et al. 1994; Glayzer et al. 1995). Transposable elements have been shown to play an important role in genome evolution in many types of organisms (see McDonald 1993 for review). This is likely to be especially true in asexual fungi, as sexual recombination does not contribute to genetic variation in these species. Although most fungal transposons have only been recently described, there already is evidence for the contribution of a transposable element (Pot3) to pathogenic variation in the clonally propagated pathogen Magnaporthe grisea (Farman et al. 1996).

In this study we report the molecular characterization of a putative transposable element, Nht1, that was cloned from the 1.6-Mb MAK1 PDA6-1 chromosome in N. haematococca MPVI isolate 156-30-6. This repeated element is highly concentrated on the chromosome from which it was cloned. Because transposable elements are associated with chromosomal aberrations in other organisms we hypothesize that Nht1 contributes to the instability of the $1.6-\mathrm{Mb}$ dispensable chromosome in isolate 156-30-6 during sexual reproduction.

\section{RESULTS}

\section{Cloning and sequence analysis of $\mathbf{N h t 1}$.}

The apparent transposable element described herein was discovered during the cloning of MAK1 from the 1.6-Mb dispensable chromosome of $N$. haematococca MPVI isolate 15630-6 (Covert et al. 1996). MAK1 was cloned from a cosmid (1F7) that hybridized to approximately $22 \%$ of the clones in a chromosome-specific cosmid library. Such a large number of positive clones suggested the presence of repeated DNA sequences on the 1F7 cosmid. In order to identify a clonable DNA fragment containing such sequences, a collection of clones that hybridized to, but did not overlap with, cosmid 1F7 was used in detailed cross-hybridization studies. Southern blots of the restriction enzyme-digested clone collection were probed independently with several representatives of the group. These experiments led to identification of a $1.3-\mathrm{kb}$ EcoRI fragment in cosmid 2D11 that hybridized to 50\% of the 1F7-homologous clones in the chromosome-specific library. This fragment was cloned and sequenced. A BLAST data base search revealed that the deduced amino acid sequence of the $N$. haematococca MPVI clone was similar to portions of two fungal transposons: Fotl of Fusarium oxysporum (Daboussi et al. 1992) and Pot2 of Magnaporthe grisea (Kachroo et al. 1994). These sequence comparisons also revealed that the 1.3$\mathrm{kb}$ EcoRI fragment from $N$. haematococca MPVI did not appear to contain the entire transposon. Additional subclones from $2 \mathrm{D} 11$, therefore, were sequenced to identify the remainder. This copy of the repeated element is named Nht1A and its complete sequence is shown in Figure 1 . The $5^{\prime}$ and $3^{\prime}$ termini of $N$ t 1 were defined by sequencing the corresponding regions of two additional copies of Nhtl, designated $N h t 1 B$ and Nht1C, on two other 1F7-homologous cosmid clones (cosmids $1 \mathrm{~A} 8$ and $2 \mathrm{H} 8$, respectively). The homology between the three Nht1 copies terminates after a TA dinucleotide at each end of the common sequences (data not shown).

NhtlA is 2,198 bp long and has incomplete ITRs of 102 and 84 bp (Fig. 1). Its $5^{\prime}$ ITR contains a direct repeat of $14 \mathrm{bp}$; its 3' ITR contains only one, imperfect copy of this repeat (Figs. 1 and 2). The ITRs of NhtlC are organized similarly (data not shown), but those of Nht1B differ in that its $3^{\prime}$ ITR contains two copies of the $14 \mathrm{bp}$ repeat (Fig. 2). The identity between Nht $1 A$ and $N h t 1 B$ at their $5^{\prime}$ and $3^{\prime}$ ITRs is 83 and $82 \%$, respectively, not including the difference caused by the missing copy of the 14-bp repeat in NhtlA (Fig. 2). Within their coding regions these two copies of $N h t 1$ are almost identical. For example, the last 1,500 bp of Nht1A are $91 \%$ identical to the corresponding bases in Nht1B (data not shown).

When the complete NhtlA amino acid sequence was compared with that of Fotl and Pot 2 the presence of two short introns in Nhtl became apparent (Fig. 1). If the introns are not spliced out prior to translation, the NhtlA sequence homologous to the transposons shifts frame twice. However, if the putative introns are spliced out, a single open reading frame homologous to Fot1 results (Fig. 3). The sequences of the $5^{\prime}$ and $3^{\prime}$ splice junctions of the Nht $1 A$ introns match known fungal splice junctions (Gurr et al. 1987). Introns are also present in the corresponding regions of Nht1B. NhtlA and NhtlB share $75 \%$ identity in the first intron and $100 \%$ identity in the second intron (data not shown).

There are two potential translation start sites in Nht1A (Fig. 1, nucleotides 298 and 340). Assuming the first start site is correct, the putative transposase encoded by NhtlA consists of 550 amino acids. Nht1A, Fotl, and Pot 2 are most similar over the first two-thirds of their proteins. The region defined by amino acids 10 to 384 of Nht1A is $48 \%$ identical to Fotl (Fig. 3) and $42 \%$ identical to Pot2. Nht1A is less similar to Pot3 and Fccl, transposable elements of the same structural family, isolated from Magnaporthe grisea and Cochliobolus carbonum, respectively (Farman et al. 1996; Panaccione et al. 1996). In the region defined by amino acids 10 to 384 of Nht1A, Pot 3 shares $30 \%$ identity and Fccl shares $32 \%$ identity with NhtlA.

\section{Copy number and geographic distribution of Nht1.}

Southern blots of isolate 156-30-6 and several N. haematococca MPVI field isolates were used to investigate the copy number and geographic distribution of Nht1. Genomic DNA from these isolates was digested with two restriction enzymes that do not cut within NhtlA, and probed with an internal fragment of Nht1A (Fig. 4A, probe A). The number of Nht 1 copies in N. haematococca MPVI varies widely. Isolates T-69, T-95, and T-110 are devoid of the transposon (Fig. 4B). These three isolates were collected in the southeastern United States (Table 1). In contrast, isolates T-30 and T-126 are estimated to have up to 100 copies (Fig. 4B). These isolates were both collected in the northwestern United States (Table 1). The remaining isolates are estimated to contain approximately 10 copies (156-30-6, T-77, T-161, and T-200) or 20 copies (T-25, T-27, and T-33) of Nht1 and do not fall into clear geographical categories (Fig. 4B and Table 1). 
CCATCCACAACCCCTCTTTCGGCATACCCCCICITTCGGCAAGCAAAATAAAAAAAGCTT CACCAAAACTCACCAACTTCACTIATACCAGAATTGGCCAAAGTAAATAGC TACTTAGAG GACAATGCCTCTATCGATTATAGAAATGATTGGAGTTAACGACTTTAGCTCTCTTAGGCT GTGCGGGGTACGCGATCTATGCATGCTGTGCTATGGAAATAGTGTTGGTTTAATCATCAA ACTCCTCACCGCTCTGITCCGTGTTTTTTGACCTTTGTCTGCGACAACGACAAGAAAATG

CCTAGgTCGAGACCTCGaTgGaAatATACAGAGgaAaACATGgCagaggCTATCTTGGA $\begin{array}{llllllllllllllllllll}P & R & S & R & P & R & W & K & Y & T & E & E & N & M & A & E & A & I & L & D\end{array}$ GTTACTGATGATGGCGITTCACCTCCTCAAGCCGCTCAGAGACGGGGAGTGCCTCGAAGC $\begin{array}{llllllllllllllllllll}V & T & D & D & G & V & S & P & P & O & A & A & Q & R & R & G & V & P & R & S\end{array}$ ACTCTAGTCGACAGACTIAACGGCCAGAAAGCCGTGAAAGAGCAGATTCAGCCTCGCCGA

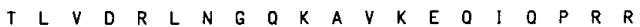
CGTTTGTCCAAGAATCAAGAGGACAGATTGGCTTTCTGGATCCTCCGTCAGGAATCTCTG

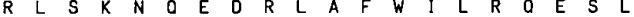
GGCTATGCTCCGTCCCACAATCAGATCCGCGCTTGCGTCACGGGCTTGTTGAGACAGCAG

$\begin{array}{llllllllllllllllllll}G & Y & A & P & S & H & N & a & \text { I } & R & A & C & V & T & G & L & L & R & a & a\end{array}$ GGCGAACATCCCGAGTTAGGACGCAATTGGGTGACTAGATTTATCAAGCGCCGCACAGAC

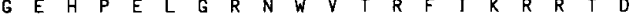
CTGACGACCAAGATGGGTAGACGCCAAGAAGCCAAAAGGTCTGACTCTTTCACGCCTAAG

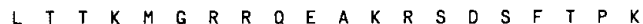
GCAGTTCATTGGTACTTTGATATCAGGGAGGGCCAGTATGGCTGGATCAAGCCTGAAAAC

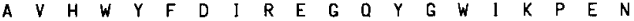
ACCGICAACGTTGACGAAGGGGGTATTATGACTGGTTTCGGTAAGCATCTATCTGTATAC

T V N V D E G G I M T G F D CTIGTCAGTGCTTGAAATTIGACCGACTAATCCTIITTATTTAGGCTTAGATAGCCTGGT TGT $L \quad D S L \quad V$

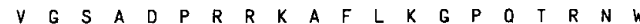
GACTTCATTTATCGAAGCTGTCACTGCTGACGGCCGCGCCTTAATCCCTGGCATAATCTT

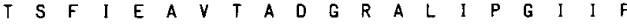
CAAGGGGAAAGAACTGCAGAAACAGTGGTTTGTTGAGGAATTCAAGGAGATAGCAGACTG

$K$ G K E L O K O W F V E E F K E I A D W GTATTACATAACTTCGCCTAACGGGTGGACTGACGACCACATTGGCGTTGAATGGCTTGA $\begin{array}{llllllllllllllllllll}Y & Y & I & T & S & P & N & G & W & T & D & D & H & I & G & V & E & W & L & E\end{array}$ AAGAGTCTATCTGCCCCAGACAATGCCGGCCGACGACTCTGATGCGCGTCTGATCATATT

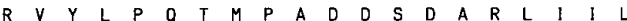
AGATGGCCATGGAAGTCATGCAACGGTATGTTCTICCTITTCCTCAGCTCAAGGTCACTA D $\quad G \quad H \quad G \quad S$ H $A$ T CTAAGTAAGGGAAGGATGAATGGATGGCCACGTGCTIITIGAATAACGTTIATTGTTGCT

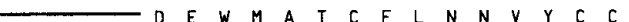
ATCTGCCAGCACACTGCTCTCATGGGCTCCAGCCGITGGACAATGGAGTATTCAATGCCT

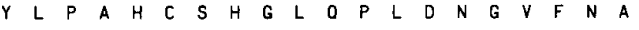
CCAAAGCCGCATATCGACGAGAGTTGGAGAATTTCGCTTCACTGACTGATICCACTCCAA

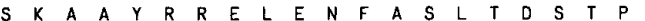
TGGACAAGGTCAATTTCATCAGGGCCTACGCTAAGGCGCGTCGAGTTGGAATGACTGAGA

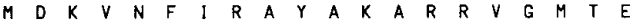
AGAACATACTCTCCGGC TGGAGGGTTACTGGGAATTGGCCGATCTCACGTGCCAAAGCGC

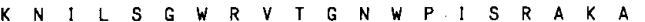
TGCCACACCCAGAAATCCAACAAGATAGGCCAAATGGCAGCCCAAGAGCGACTCCCGAAC $L$ P $H$ P P E I O CCAGGCCGTATTTTGACTCGGACGATACACCAAAGACGAGCCGTCAAATICGTGATCTIG $\begin{array}{llllllllllllllllllll}P & R & P & Y & F & D & S & D & D & T & P & K & T & S & R & Q & I & R & D & L\end{array}$ GGCTGAACAAAACACCAAAGACACGAAGACGGTATAACGTAATAGCAAAGGGCTITGAGG $\begin{array}{llllllllllllllllllll}G & L & N & K & T & P & K & T & R & R & R & Y & N & V & I & A & K & G & F & E\end{array}$ CTCAACAGCAGACGGTAGCGGCGCATACTGCGAGGATTGCTAGCCTAGAGGAGGAATTGG

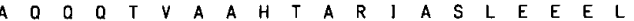
CTCGCCTGAAGAGAGGGAAGAAGAGGAAGGCGGTGCCGAATCCTAACAAACGTTTTATGA

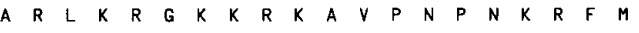
CTCTTGGTGAGACTCTAGCTGCTGGGGAAGCCATATCCGAAGAGGAGACTCAAAATATGC T L G E CTGTTGCGGTGGAATGTGGCCGITCAGGGGAGCCGGGGTCAGAATCGGAGGCTGCGTCGG

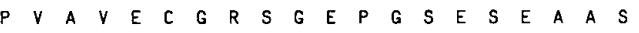
TCATTGAAGTCAGAGAGGAAACCATACCCCATCAACTAACCACGCGGTCAGGGCGGCTTA $\begin{array}{llllllllllllllllllll}V & I & E & V & R & E & E & T & I & P & H & O & L & T & T & R & S & G & R & L\end{array}$ TCAAAAGACCCAGgATTCAATAGAGAGATGCAACTATTATTCACAAGACGCAAATICITG $\begin{array}{lllllllll} & K & R & P & R & 1 & 0\end{array}$ CACGCGCCTATCAAITTGGCCAAGITCTGTGTAAGTAAACATGITGATTTITGGTGGTGC IITTIITTITGCCTGGCGAAGGGTGGTGTGCATGG 2198

Fig. 1. Nucleotide sequence of Nht1A. Deduced amino acid sequence is shown below the DNA sequence in one letter code. Putative introns are indicated by a solid line. Terminal inverted repeats are underlined with a dashed line and tandem repeats are overlined with arrows.

\section{Distribution of Nht1 within the genome.}

To investigate the genomic distribution of Nhtl, the chromosomal DNA of isolate 156-30-6 was separated by contourclamped homogeneous electric field (CHEF) electrophoresis (Fig. 5, lane 1) and probed with an internal fragment of Nht1A (Fig. 4A, probe A). Four chromosomal bands hybridized to this probe (Fig. 5, lane 2). Each band was quantified with a PhosphorImager (Molecular Dynamics, Sunnyvale, CA) to compare the relative intensities of their hybridization signals. The most intense hybridization signal corresponds to the 1.6Mb chromosome from which NhtlA was cloned. The hybridization signals from the 2.5- and 3.4-Mb chromosomal bands are 5 to 6 times less intense than that of the 1.6-Mb band. The weakest hybridization signal corresponds to a $5.0-\mathrm{Mb}$ chromosomal band; it is 19 times less intense than that of the 1.6$\mathrm{Mb}$ band and is not clearly visible in the exposure displayed in Figure 5. This weak signal appears to be partially due to the fact that the 5.0-Mb band contains a lower concentration of chromosomal molecules than the smaller bands in the gel (Fig. 5, lane 1). An ongoing physical mapping project in our laboratory suggests that the $1.6-\mathrm{Mb}$ chromosome in isolate 156-30-6 contains at least six copies of Nht1 (data not shown). Based on this information and the relative intensity of the hybridization signals shown in Figure 5, the 2.5- and 3.4-Mb hybridization signals are estimated to indicate the presence of one to two copies of Nhtl per chromosomal band. One copy of Nhtl is also estimated to be present on the $5.0-\mathrm{Mb}$ band. Therefore, in total, isolate 156-30-6 contains nine to 11 copies of Nhtl in its genome, a number that corresponds well to the copy number estimated from the Southern blot in Figure 4. Out of these nine to 11 copies, most are concentrated on the 1.6-Mb dispensable chromosome.

CHEF-separated DNA of isolate 156-30-6 was also probed with a flanking fragment of Nht1A to confirm that Nht1A was cloned from the 1.6-Mb dispensable chromosome. The 1.6$\mathrm{Mb}$ chromosome was the only positive band with this probe (Fig. 5A, lane 3).

To examine the genomic distribution of Nht1 in the parents of 156-30-6, a Southern blot of T-126 and T-161 CHEFseparated DNA was probed with an internal fragment of Nht1A (Fig. 5B). The distribution of Nhtl in the T-161 genome resembles its distribution in 156-30-6; multiple Nht copies appear to be on a $1.6-\mathrm{Mb}$ chromosome and a few copies are on larger chromosomes. T-126 is very different in that it has several chromosomes in the range of 1.0 to $3.5 \mathrm{Mb}$ that contain multiple copies of $N h t 1$. On long exposures $N h t 1$ also appears to be present on some of the large chromosomes (>5 $\mathrm{Mb}$ ) of isolate T-126 (data not shown). As in 156-30-6, interpretation of hybridization intensity in T-126 and T-161 is confounded by the fact that the largest chromosomes $(>4.5 \mathrm{Mb})$ are under-represented relative to the smaller chromosomes on CHEF gels.

\section{DISCUSSION}

We conclude Nhtl is a transposon because of its repeated nature and structural similarity to previously described fungal transposons. This particular family of transposable elements possesses two short direct repeats embedded within longer ITRs. The ITRs flank a coding sequence for a putative transposase. The presence of TA dinucleotides on either end of 
Nht1 suggests TA is a duplicated target site for this element as was demonstrated for Fot1 (Daboussi et al. 1992) and Pot2 (Kachroo et al. 1994). However, the ITRs of Nht1 are about 2.5 times longer than those of Fot1, Pot2, and Pot 3 and about 1.5 times longer than those of Fccl. Also, the coding sequence of Nhtl appears to contain two introns whereas these other transposable elements lack introns.
The structure of the three cloned copies of Nht1 is not uniform. The differences are most evident in the ITRs. NhtlB contains two complete sets of a 14-bp direct repeat in its ITRs. Nht1A and Nht1C, however, both lack a copy of the direct repeat in their $3^{\prime}$ ITRs. This raises the possibility that Nhtl is able to transpose without having complete ITRs. Alternatively, multiple copies of the element with the truncated $3^{\prime}$
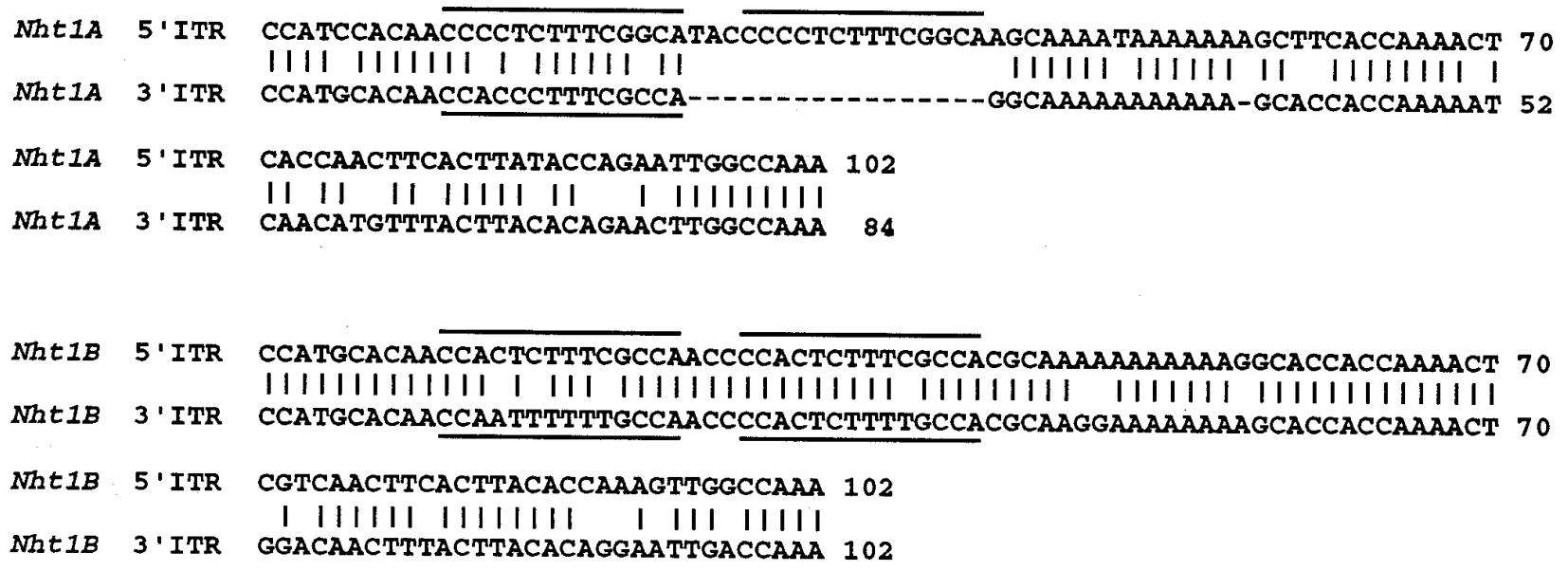

Fig. 2. Alignments of $5^{\prime}$ inverted terminal repeats (ITRs) and $3^{\prime}$ reverse-complement ITRs from $N h t 1 A$ and $N h t 1 B$. Identical nucleotides are indicated with vertical lines and tandem repeats are over-lined or under-lined. Dashes represent gaps introduced to improve alignments.

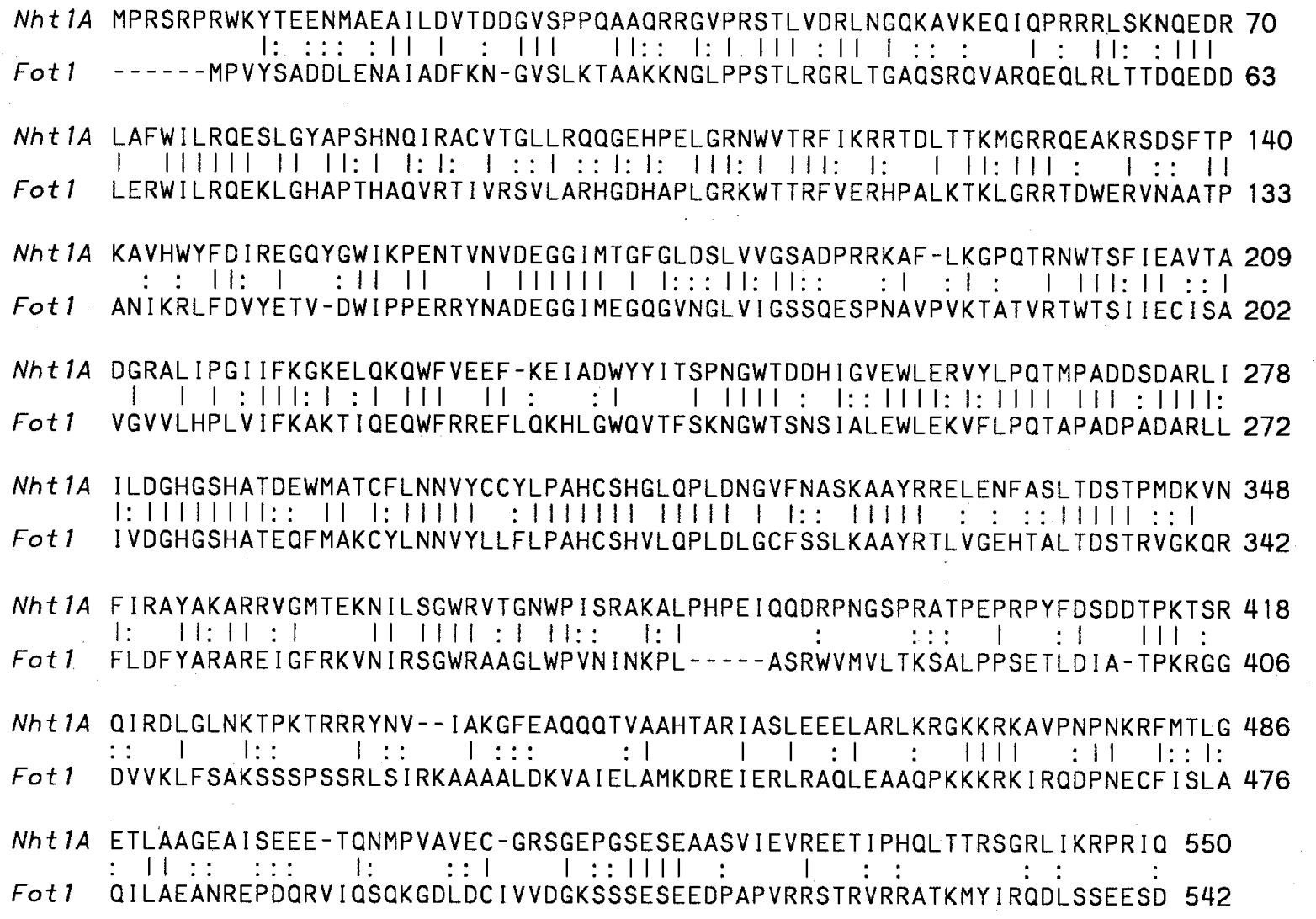

Fig. 3. Amino acid alignment of putative transposases from Nht1A and Fot1. Bars represent identical amino acids and dots indicate a frequently observed amino acid change, according to the PAM 250 matrix (Dayhoff et al. 1978). 
ITR might have arisen from recombination events. The single cloned copy of $F c c l$ also lacks the second direct repeat in its 3' ITR (Panaccione et al. 1996). This copy of Fccl, however, is thought to be inactive because of the incomplete $3^{\prime}$ ITR and because its putative transposase open reading frame is interrupted by a stop codon.

Whether or not Nhtl is an active transposon in any of the $N$. haematococca MPVI isolates that contain it remains an open question. Processes (named RIP and MIP) that inactivate repeated sequences during sexual reproduction have been well characterized in Neurospora crassa and Ascobolus immersus (Cambareri et al. 1989; Goyon and Faugeron 1989). In addi-

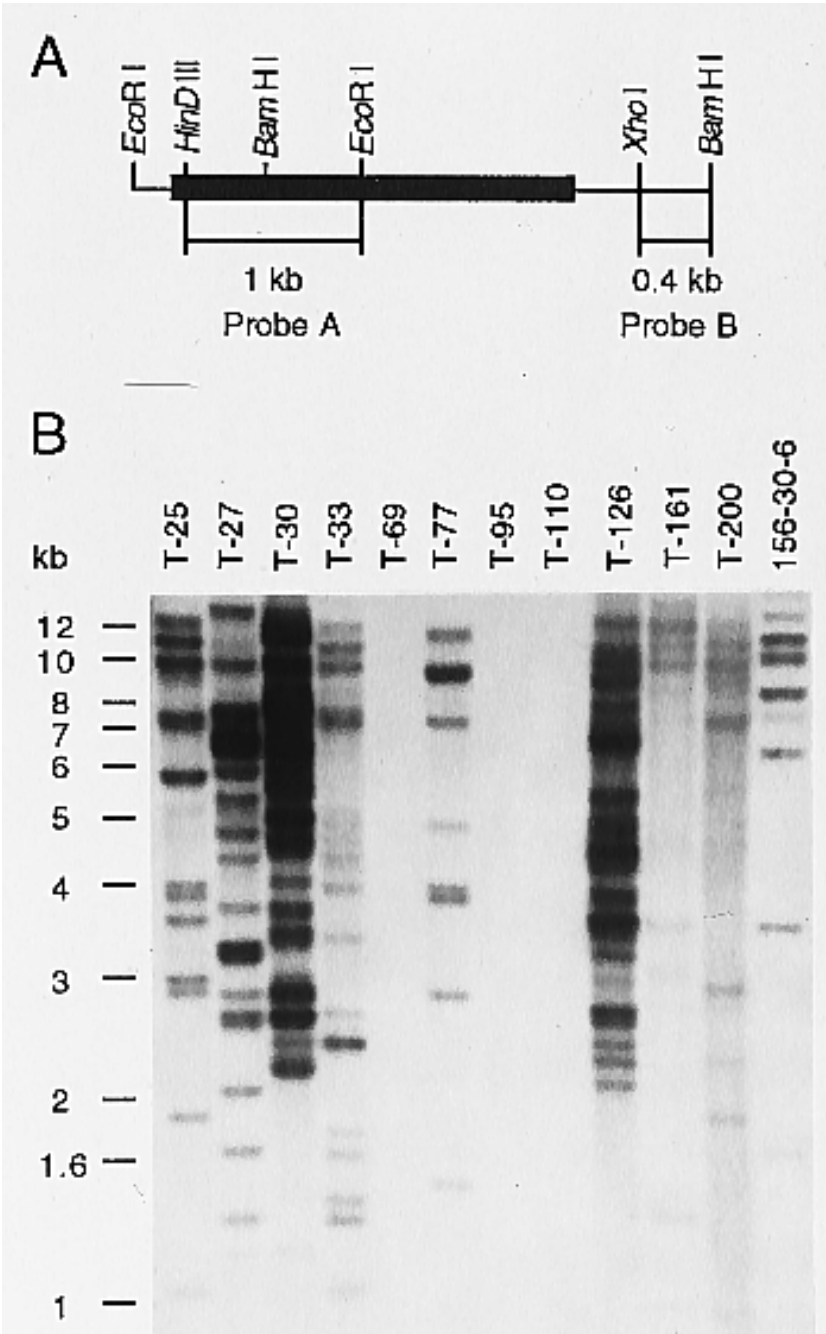

Fig. 4. Presence of Nht1 in various Nectria haematococca mating population VI isolates. A, Restriction map of Nht1A and flanking DNA. NhtlA is indicated as a thick black bar. B, Equal amounts of genomic DNA $(3 \mu \mathrm{g})$ were double digested with $K p n I$ and $X b a I$, separated on a $0.8 \%$ agarose gel, and Southern blotted. The corresponding Southern blot was probed with an internal fragment of Nht1A (A, probe A). Molecular weight standards are indicated on the left in kilobases $(\mathrm{kb})$. tion, inactivation of multiple copy transgenes has been observed in sexual progeny of Gibberella fujikuroi (Leslie and Dickman 1991). Consequently, repeated transposable elements in any of these three ascomycetes would probably be rendered immobile if put through a cross. Although it is not known if similar inactivation processes exist in $N$. haematococca MPVI, these data from related fungi raise the possibility that all of the Nht1 copies in isolate 156-30-6 were inactivated during the cross that produced 156-30-6. Future work on the mobility of Nhtl will need to be carried out with this possibility in mind.

The number of Nhtl-homologous bands in N. haematococca MPVI field isolates from different locations in North America is highly variable. For example, of the 11 isolates examined here, three completely lack Nht1 while two others each appear to have about 100 copies. Our survey did not include enough isolates to determine if there is a correlation

Table 1. Strains of Nectria haematococca mating population VI used in this study

\begin{tabular}{llll}
\hline Isolate & Origin & Habitat & Reference \\
\hline T-25 & New York, USA & Chickpea & VanEtten 1978 \\
T-27 & California, USA & Chickpea & VanEtten 1978 \\
T-30 & Washington, USA & Pea & VanEtten 1978 \\
T-33 & Canada & Cottonwood & VanEtten 1978 \\
T-69 & South Carolina, USA & Tuliptree & VanEtten 1978 \\
T-77 & Pennsylvania, USA & Alfalfa & VanEtten 1978 \\
T-95 & South Carolina, USA & Tuliptree & VanEtten 1978 \\
T-110 & Louisiana, USA & Cottonwood & VanEtten 1978 \\
T-126 & Montana, USA & Sainfoin & VanEtten 1978 \\
T-161 & Unknown & Unknown & Lucy et al. 1988 \\
T-200 & Unknown & Red clover & Lucy et al. 1988 \\
156-30-6 & Progeny isolate of & $\ldots$ & Miao and VanEtten \\
& cross T-126 $\times$ T-161 & & 1992a \\
\hline
\end{tabular}

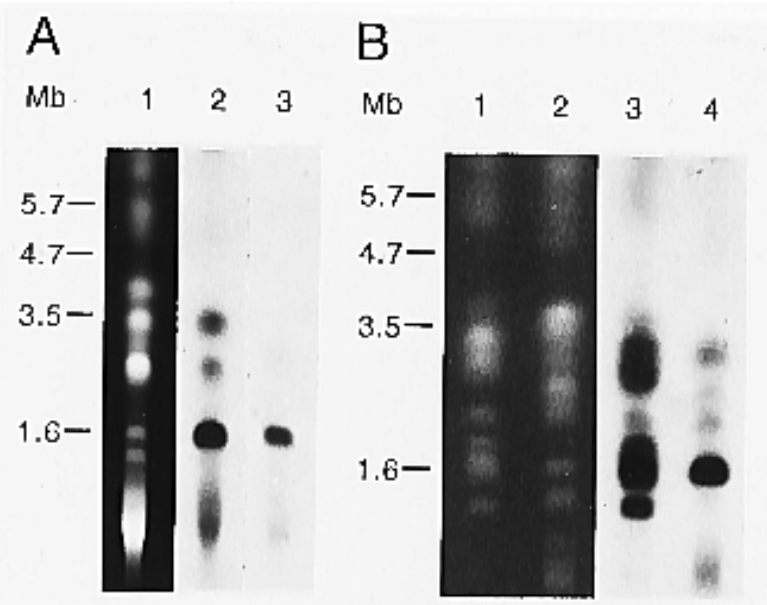

Fig. 5. Intragenomic distribution of Nht1 and chromosomal location of Nht1A. A, Lane 1: Ethidium bromide-stained contour-clamped homogeneous electric field (CHEF) gel of isolate 156-30-6. Lane 2: Southern blot of lane 1 hybridized to an internal fragment of Nht1A (Fig. 4A, probe A). Lane 3: Southern blot of 156-30-6 DNA, separated as in lane 1, hybridized to DNA flanking Nht1A (Fig. 4A, probe B). B, Ethidium bromide-stained CHEF gel of field isolates. Lane 1: T-126; Lane 2: T161; Lanes 3 and 4: Southern blot corresponding to lanes 1 and 2 hybridized to an internal fragment of Nht1A (Fig. 4A, probe A). A and B, Molecular weight markers (Schizosaccharomyces pombe chromosomes) and the 1.6-Mb dispensable chromosome are indicated on the left of each panel in $\mathrm{Mb}$. 
between habitat (host plant) and presence or absence of Nht1; however, our data does suggest there is an association between geographical location and Nhtl distribution. All three $N$. haematococca MPVI isolates lacking the transposon were collected in the southeastern United States, and both isolates containing the highest Nhtl copy number were collected in the northwestern United States. Maruyama and Hartl (1991a) proposed two hypotheses to explain a discontinuous distribution of transposable elements within one species. One model proposes that the transposon was present in a common ancestor, but has been lost over time by individual lineages. The second model proposes a recent introduction of the element by interspecific transfer. To test the applicability of these models to Nht1, a more detailed phylogenetic study of the N. haematococca MPVI field isolate collection is required.

The distribution of Fot 1, Pot2, Pot3, and Fccl has also been investigated in the fungal species from which they were isolated. Fotl is present in most $F$. oxysporum isolates as well as a few isolates of different Fusarium spp., such as Fusarium solani (Daboussi and Langin 1994). The Pot3 element in M. grisea appears to be distributed in a host-specific manner; it is present in high copy number in rice pathogens and rare or absent in non-rice pathogens (Hamer et al. 1989; Farman et al. 1996). In contrast, the Pot 2 element of M. grisea appears to be distributed rather homogeneously between isolates from different host plants and also between different geographical regions (Kachroo et al. 1994). $F c c l$ is present at least in three $C$. carbonum races and two other Cochliobolus spp. (Panaccione et al. 1996).

In field isolate T-161, most copies of Nhtl are present on a single chromosome. This distribution of Nhtl contrasts with the even distribution of Pot 2 and Pot 3 across all of the chromosomes in M. grisea (Talbot et al. 1993; Kachroo et al. 1994; Orbach et al. 1996). The uneven distribution of Nhtl in $\mathrm{T}-161$ could be the result of more than one evolutionary process. It is possible that when Nhtl was first introduced in T-161 it inserted by chance into the $1.6-\mathrm{Mb}$ chromosome and since then has transposed primarily within this chromosome. Such an idea is not without precedent, as a preference for short distance transposition has been demonstrated for the $A c$ element in Zea mays (Van Schaik and Brink 1959; Dooner and Belachew 1989) and the $P$ element in Drosophila (Tower et al. 1993; Zhang and Spradling 1993). Another possibility is that the chromosome containing multiple copies of Nhtl was added intact to the T-161 genome, perhaps by crossing with an isolate containing Nht1 throughout its genome, or via horizontal transfer from another species. The horizontal transfer of genetic information between different fungal species, or between genetically isolated lines of the same species, has been proposed to occur in C. carbonum (Nikolskaya et al. 1995) and Colletotrichum gloeosporioides (Masel et al. 1996). Horizontal transfer of transposable elements has also been documented to occur between species of the Drosophilidae (Daniels et al. 1990; Maruyama and Hartl 1991b). However, considering the potential for sexual reproduction in $N$. haematococca MPVI and the existence of field isolates containing many copies of Nhtl, transfer from another species does not appear to be the most likely explanation for the introduction of Nht1 into T-161. Regardless of how Nhtl was introduced into T-161, its limited intragenomic distribution suggests either that it was introduced relatively recently, or that it became inactive shortly after its introduction, or that it transposes only infrequently.

Our current understanding of how chromosome-length polymorphisms arise and are maintained in fungi is limited (Zolan 1995). The rearrangements of chromosomes observed in $C$. cinereus and L. maculans have been explained as the result of recombination between homologous chromosomes of unequal length (Zolan et al. 1994; Plummer and Howlett 1995), but direct evidence in support of this hypothesis in either case has not yet been reported. In $N$. haematococca MPVI, chromosomal instability has been observed even when homologues of equal size are paired (Miao et al. 1991a), and the resulting chromosome sizes suggest that intrachromosomal recombination is at work. A better understanding of chromosomal rearrangements in fungi will contribute not only to our general knowledge of fungal genome evolution, but also is likely to contribute to our understanding of pathogenic variation in organisms capable of causing disease. Transposable elements are likely agents in these processes, as they stimulate chromosomal aberrations such as translocations, deletions, and inversions in other eukaryotes (Roeder and Fink 1980; Engels and Preston 1984; Rothstein et al. 1987; Lim 1988; Döring et al. 1990). Therefore, the discovery of Nhtl in many copies on an unstable chromosome in $N$. haematococca MPVI may be an important step toward elucidation of the mechanisms underlying chromosomal instability in this organism.

\section{MATERIALS AND METHODS}

\section{Fungal isolates and plasmids.}

The Nectria haematococca MPVI isolates used in this study are listed in Table 1. Field isolates are prefaced by the letter "T" and were provided by Hans VanEtten, University of Arizona. All isolates were grown and maintained on V8 agar medium (Miller 1955) at $28^{\circ} \mathrm{C}$ in the dark.

\section{DNA manipulations.}

Standard molecular biology techniques were used throughout (Sambrook et al. 1989). The cosmid clones used in this study are from a genomic library of 156-30-6 (Covert et al. 1996) in the cosmid vector pMOcosX (Orbach 1994). In the version of the library used previously, cosmid 1F7 was named 1G8 (Covert et al. 1996). All subclones from cosmid 2D11 were made in Bluescript II SK ${ }^{+}$(Stratagene, La Jolla, CA). Fungal DNA was isolated as previously described (Covert et al. 1996). Gels were blotted onto Hybond $\mathrm{N}^{+}$membranes (Amersham, Arlington Heights, IL). All hybridization reactions were carried out according to the aqueous solution protocol described in Ausubel et al. (1987) at $65^{\circ} \mathrm{C}$. Probes were synthesized with the DECAprime II DNA labeling Kit (Ambion, Austin, TX). Blots were washed at $65^{\circ} \mathrm{C}$ twice with $2 \times \mathrm{SSC}\left(1 \times \mathrm{SSC}=0.15 \mathrm{M} \mathrm{NaCl}, 0.015 \mathrm{M} \mathrm{Na}_{3}\right.$ citrate, $\mathrm{pH} 7.0$ with $\mathrm{HCl}), 0.1 \%$ sodium dodecyl sulfate (SDS) and once with $0.5 \times$ SSC, $0.1 \%$ SDS. Agarose gels were scanned with an Eagle Eye II gel scanner (Stratagene) to measure band intensity, and hybridization signals were quantified with a PhosphorImager (Molecular Dynamics, Sunnyvale, CA).

To prepare chromosomal DNA for pulsed-field gel electrophoresis the protoplasting protocol described by Powell and Kistler (1990) was used with a few modifications. Glucanex (Novo Nordisk Ferment AG, Dittingen, Switzerland) was used 
as the protoplasting enzyme at a concentration of $3.75 \mathrm{mg} / \mathrm{ml}$ in $1.2 \mathrm{M} \mathrm{MgSO}_{4}, 50 \mathrm{mM}$ sodium citrate $\mathrm{pH}$ 5.8. Protoplasts were embedded and lysed as described by (Miao et al. 1991b). Chromosomal sized DNA was separated in $0.8 \%$ agarose gels (low EEO grade, Fisher Scientific, Pittsburgh, PA) in 1× TAE (0.04 M Tris-acetate, 0.001 M EDTA) on a CHEF DR III unit (BioRad, Hercules, CA). The separation was carried out at $14^{\circ} \mathrm{C}$ for $72 \mathrm{~h}$ at $2 \mathrm{~V} / \mathrm{cm}$ with ramped switch intervals from 1,200 to 2,400 s. Schizosaccharomyces pombe and Saccharomyces cerevisiae (FMC Bioproducts, Rockland, ME) were loaded on each gel as size markers.

\section{DNA sequence analysis.}

All nucleotide sequencing was carried out on an PE Applied Biosystems sequencer (Foster City, CA) at the Molecular Genetics Instrumentation Facility at the University of Georgia, Athens. A mixed strategy of sequencing subclones with T3 and T7 primers, or with Nhtl-specific, synthesized primers, was applied to sequence both strands of NhtlA. The same synthesized primers were used to sequence the ends and internal portions of $N h t 1 B$ and NhtlC. BLAST (Altschul et al. 1990) and the DNASTAR software package (DNASTAR, Madison, WI) were used to analyze, translate, and compare DNA sequences.

\section{ACKNOWLEDGMENTS}

We thank Patton Hilliard for technical assistance and Scott Gold for providing critical comments on the manuscript. The work described in this paper was supported by the National Science Foundation under grant no. MCB-9507263.

\section{LITERATURE CITED}

Altschul, S. F., Gish, W., Miller, W., Myers, E. W., and Lipman, D. J. 1990. Basic local alignment search tool. J. Mol. Biol. 215:403-410.

Ausubel, F. M., Brent, R., Kingston, R. E., Moore, D. D., Seidman, J. G., Smith, J. A., and Struhl, K. 1987. Current Protocols in Molecular Biology. John Wiley \& Sons, New York.

Cambareri, E. B., Jensen, B. C., Schabtach, E., and Selker, E. U. 1989. Repeat-induced G-C to A-T mutations in Neurospora. Science 244: 1571-1575.

Ciuffetti, L. M., and VanEtten, H. D. 1996. Virulence of a pisatin demethylase-deficient Nectria haematococca MPVI isolate is increased by transformation with a pisatin demethylase gene. Mol. PlantMicrobe Interact. 9:787-792.

Covert, S. F., Enkerli, J., Miao, V. P. W., and VanEtten, H. D. 1996. A gene for maackiain detoxification from a dispensable chromosome of Nectria haematococca. Mol. Gen. Genet. 251:397-406.

Daboussi, M. J., and Langin, T. 1994. Transposable elements in the fungal plant pathogen Fusarium oxysporum. Genetica 93:49-59.

Daboussi, M. J., Langin, T., and Brygoo, Y. 1992. Fot1, a new family of fungal transposable elements. Mol. Gen. Genet. 232:12-16.

Daniels, S. B., Peterson, K. R., Strausbaugh, L. D., Kidwell, M. G., and Chovnick, A. 1990. Evidence for horizontal transmission of the $P$ element between Drosophila species. Genetics 124:339-355.

Dayhoff, M. O., Schwartz, R. M., and Orcutt, B. C. 1978. A model of evolutionary change in proteins. Pages 345-352 in: Atlas of Protein Sequence and Structure. M. O. Dayhoff, ed. National Biomedical Research Foundation, Washington, DC.

Dobinson, K. F., Harris, R. E., and Hamer, J. E. 1993. Grasshopper, a long terminal repeat (LTR) retroelement in the phytopathogenic fungus Magnaporthe grisea. Mol. Plant-Microbe Interact. 6:114-126.

Dooner, H. K., and Belachew, A. 1989. Transposition pattern of the maize element $A c$ from the $b z-m 2(A c)$ allele. Genetics 122:447-457.

Döring, H.-P., Pahl, I., and Durany, M. 1990. Chromosomal rearrangements caused by the aberrant transposition of double $D S$ elements are formed by $D s$ and adjacent non- $D s$ sequences. Mol. Gen. Genet. 224: 40-48.

Engels, W. R., and Preston, C. R. 1984. Formation of chromosome rearrangements by $P$ factors in Drosophila. Genetics 107:657-678.

Farman, M. L., Taura, L., and Leong, S. A. 1996. The Magnaporthe grisea DNA fingerprinting probe MGR586 contains the $3^{\prime}$ end of an inverted repeat transposon. Mol. Gen. Genet. 251:675-681.

Finnegan, D. J. 1989. Eukaryotic transposable elements and genome evolution. Trends Genet. 5:103-107.

Glayzer, D. C., Roberts, I. N., Archer, D. B., and Oliver, R. P. 1995. The isolation of Ant1, a transposable element from Aspergillus niger. Mol. Gen. Genet. 249:432-438.

Goyon, C., and Faugeron, G. 1989. Targeted transformation of Ascobolus immersus and de novo methylation of the resulting duplicated DNA sequences. Mol. Cell. Biol. 9:2818-2827.

Gurr, S. J., Unkles, S. E., and Kinghorn, J. R. 1987. The structure and organization of nuclear genes of filamentous fungi. Pages 93-139 in: Gene Structure in Eukaryotic Microbes. J. R. Kinghorn, ed. IRL Press, Oxford.

Hamer, J. E., Farrall, L., Orbach, M. J., Valent, B., and Chumley, F. G. 1989. Host species-specific conservation of a family of repeated DNA sequences in the genome of a fungal plant pathogen. Proc. Natl. Acad. Sci. USA 86:9981-9985.

Kachroo, P., Leong, S. A., and Chattoo, B. B. 1994. Pot2, an inverted repeat transposon from the rice blast fungus Magnaporthe grisea. Mol. Gen. Genet. 245:339-348.

Kinsey, J. A., and Helber, J. 1989. Isolation of a transposable element from Neurospora crassa. Proc. Natl. Acad. Sci. USA 86:1929-1933.

Kistler, H. C., Meinhardt, L. W., and Benny, U. 1996. Mutants of Nectria haematococca created by a site-directed chromosome breakage are greatly reduced in virulence toward pea. Mol. Plant-Microbe Interact. 9:804-809.

Leslie, J. F., and Dickman, M. B. 1991. Fate of DNA encoding hygromycin resistance after meiosis in transformed strains of Gibberella fujikuroi (Fusarium moniliforme). Appl. Environ. Microbiol. 57: 1423-1429.

Lim, J. K. 1988. Intrachromosomal rearrangements mediated by hobo transposons in Drosophila melanogaster. Proc. Natl. Acad. Sci. USA 85:9153-9157.

Lucy, M., Mathews, P., and VanEtten, H. D. 1988. Metabolic detoxification of the phytoalexins maackiain and medicarpin by Nectria haematococca field isolates: Relationship to virulence on chickpea. Physiol. Mol. Plant Pathol. 33:187-199.

Maruyama, K., and Hartl, D. L. 1991a. Evolution of the transposable element mariner in Drosophila species. Genetics 128:319-329.

Maruyama, K., and Hartl, D. L. 1991b. Interspecific transfer of the transposable element mariner between Drosophila and Zaprionus. J. Mol. Evol. 33:514-524.

Masel, A. M., He, C., Poplawski, A. M., Irwin, J. A. G., and Manners, J. M. 1996. Molecular evidence for chromosome transfer between biotypes of Colletotrichum gloeosporioides. Mol. Plant-Microbe Interact. 9:339-348.

Masel, A. M., Irwin, J. A. G., and Manners, J. M. 1993a. DNA addition or deletion is associated with a major koryotype polymorphism in the fungal phytopathogen Colletotrichum gloeosporioides. Mol. Gen. Genet. 237:73-80.

Masel, A. M., Irwin, J. A. G., and Manners, J. M. 1993b. Minichromosomes of Colletotrichum spp. infecting several host species in various countries. Mycol. Res. 97:852-856.

Masel, A. M., Struijk, N., McIntyre, C. L., Irwin, J. A. G., and Manners, J. M. 1993c. A strain-specific cyclin homolog in the fungal pathogen Colletotrichum gloeosporioides. Gene 133:141-145.

McDonald, J. F., ed. 1993. Transposable elements and evolution. Special issue of Genetica 86. Kluwer Academic Pub., Dordrecht, The Netherlands.

Miao, V. P., Covert, S. F., and VanEtten, H. D. 1991a. A fungal gene for antibiotic resistance on a dispensable ("B") chromosome. Science 254:1773-1776.

Miao, V. P. W., Matthews, D. E., and VanEtten, H. D. 1991b. Identification and chromosomal locations of a family of cytochrome P-450 genes for pisatin detoxification in the fungus Nectria haematococca. Mol. Gen. Genet. 226:214-223.

Miao, V. P. W., and VanEtten, H. D. 1992a. Three genes for metabolism of the phytoalexin maackiain in the plant pathogen Nectria haemato- 
cocca: Meiotic instability and relationship to a new gene for pisatin demethylase. Appl. Environ. Microbiol. 58:801-808.

Miao, V. P. W., and VanEtten, H. D. 1992b. Genetic analysis of the role of phytoalexin detoxification in virulence of the fungus Nectria haematococca on chickpea (Cicer arietinum). Appl. Environ. Microbiol. 58:809-814.

Miller, P. M. 1955. V-8 juice agar as a general-purpose medium for fungi and bacteria. Phytopathology 45:461-463.

Nikolskaya, A. N., Panaccione, D. G., and Walton, J. D. 1995. Identification of peptide synthetase-encoding genes from filamentous fungi producing host-selective phytotoxins or analogs. Gene 165:207-211.

Orbach, M. J. 1994. A cosmid with a HyR marker for fungal library construction and screening. Gene 150:159-162.

Orbach, M. J., Chumley, F. G., and Valent, B. 1996. Electrophoretic karyotypes of Magnaporthe grisea pathogens of diverse grasses. Mol. Plant-Microbe Interact. 9:261-271.

Panaccione, D. G., Pitkin, J. W., Walton, J. D., and Annis, S. L. 1996. Transposon-like sequences at the TOX2 locus of the plant-pathogenic fungus Cochliobolus carbonum. Gene 176:103-109.

Plummer, K. M., and Howlett, B. J. 1993. Major chromosomal length polymorphisms are evident after meiosis in the phytopathogenic fungus Leptosphaeria maculans. Curr. Genet. 24:107-113.

Plummer, K. M., and Howlett, B. J. 1995. Inheritance of chromosomal length polymorphisms in the ascomycete Leptosphaeria maculans. Mol. Gen. Genet. 247:416-422.

Powell, W. A., and Kistler, H. C. 1990. In vivo rearrangement of foreign DNA by Fusarium oxysporum produces linear self-replicating plasmids. J. Bacteriol. 172:3163-3171.

Reimmann, C., and VanEtten, H. D. 1994. Cloning and characterization of the PDA6-1 gene encoding a fungal cytochrome P-450 which detoxifies the phytoalexin pisatin from garden pea. Gene 146:221-226.

Roeder, G. S., and Fink, G. R. 1980. DNA rearrangements associated with a transposable element in yeast. Cell 21:239-249.

Rothstein, R., Helms, C., and Rosenberg, N. 1987. Concerted deletions and inversions are caused by mitotic recombination between delta sequences in Saccharomyces cerevisiae. Mol. Cell. Biol. 7:1198-1207.

Sambrook, J., Fritsch, E. F., and Maniatis, T. A. 1989. Molecular Cloning: A Laboratory Manual. 2nd ed. Cold Spring Harbor Laboratory, Cold Spring Harbor, NY.

Talbot, N. J., Salch, Y. P., Ma, M., and Hamer, J. E. 1993. Karyotypic variation within clonal lineages of the rice blast fungus, Magnaporthe grisea. Appl. Environ. Microbiol. 59:585-593.

Tower, J., Karpen, G. H., Craig, N., and Spradling, A. C. 1993. Preferential transposition of Drosophila $P$ elements to nearby chromosomal sites. Genetics 133:347-359.

Tzeng, T.-H., Lyngholm, L. K., Ford, C. F., and Bronson, C. R. 1992. A restriction fragment length polymorphism map and electrophoretic karyotype of the fungal maize pathogen Cochliobolus heterostrophus. Genetics 130:81-96.

Van Schaik, N. W., and Brink, R. A. 1959. Transposition of modulator, a component of the variegated pericarp allele in maize. Genetics 44: 725-738.

VanEtten, H. D. 1978. Identification of additional habitats of Nectria haematococca mating population VI. Phytopathology 68:1552-1556.

VanEtten, H. D., Funnell-Baerg, D., Wasmann, C., and McCluskey, K. 1994. Location of pathogenicity genes on dispensable chromosomes in Nectria haematococca MPVI. Antonie Van Leeuwenhoek 65:263267.

VanEtten, H. D., Matthews, D. E., and Matthews, P. S. 1989a. Phytoalexin detoxification: Importance for pathogenicity and practical implications. Annu. Rev. Phytopathol. 27:143-164.

VanEtten, H. D., Matthews, D. E., Matthews, P. S., Miao, V., Maloney, A., and Straney, D. 1989b. A family of genes for phytoalexin detoxification in the plant pathogen Nectria haematococca. Pages 219-228 in: Signal Molecules in Plants and Plant-Microbe Interactions. B. Lugtenberg, ed. Springer-Verlag, Berlin.

Wasmann, C. C., and VanEtten, H. D. 1996. Transformation-mediated chromosome loss and disruption of a gene for pisatin demethylase decrease the virulence of Nectria haematococca on pea. Mol. PlantMicrobe Interact. 9:793-803.

Weltring, K.-M., Turgeon, B. G., Yoder, O. C., and VanEtten, H. D. 1988. Isolation of a phytoalexin-detoxification gene from the plant pathogenic fungus Nectria haematococca by detecting its expression in Aspergillus nidulans. Gene 68:335-344.

Zhang, P., and Spradling, A. C. 1993. Efficient and dispersed local $P$ element transposition from Drosophila females. Genetics 133:361373.

Zolan, M. E. 1995. Chromosome-length polymorphism in fungi. Microbiol. Rev. 59:686-698.

Zolan, M. E., Heyler, N. K., and Stassen, N. Y. 1994. Inheritance of chromosome-length polymorphisms in Coprinus cinereus. Genetics 137:87-94. 